\title{
Exploration and exploitation of the environment for novel specialized metabolites Catarina Loureiro ${ }^{1}$, Marnix H Medema ${ }^{2}$, John van der Oost ${ }^{1}$ and Detmer Sipkema ${ }^{1}$
}

\begin{abstract}
Microorganisms are Nature's little engineers of a remarkable array of bioactive small molecules that represent most of our new drugs. The wealth of genomic and metagenomic sequence data generated in the last decade has shown that the majority of novel biosynthetic gene clusters (BGCs) is identified from cultivationindependent studies, which has led to a strong expansion of the number of microbial taxa known to harbour BGCs. The large size and repeat sequences of BGCs remain a bioinformatic challenge, but newly developed software tools have been created to overcome these issues and are paramount to identify and select the most promising BGCs for further research and exploitation. Although heterologous expression of BGCs has been the greatest challenge until now, a growing number of polyketide synthase (PKS) and non-ribosomal peptide synthetase (NRPS)-encoding gene clusters have been cloned and expressed in bacteria and fungi based on techniques that mostly rely on homologous recombination. Finally, combining ecological insights with state-of-the-art computation and molecular methodologies will allow for further comprehension and exploitation of microbial specialized metabolites.
\end{abstract}

\begin{abstract}
Addresses
${ }^{1}$ Wageningen University \& Research, Laboratory of Microbiology, Stippeneng 4, 6708 WE Wageningen, The Netherlands

${ }^{2}$ Wageningen University \& Research, Bioinformatics Group,

Droevendaalsesteeg 1, 6708 PB Wageningen, The Netherlands
\end{abstract}

Corresponding author: Sipkema, Detmer (detmer.sipkema@wur.nl)

Current Opinion in Biotechnology 2018, 5:206-213

This review comes from a themed issue on Environmental biotechnology

Edited by Mike Jetten and Irene Sanchez Andrea

https://doi.org/10.1016/j.copbio.2018.01.017

0958-1669/@ 2018 Elsevier Ltd. All rights reserved.

\section{Introduction}

Microorganisms are unparalleled with respect to the chemical diversity of specialized metabolites they produce. These encompass many chemical classes including polyketides (PKs), non-ribosomal peptides (NRPs), ribosomally synthesized and post translationally modified peptides (RiPPs), terpenes, saccharides and alkaloids $\left[1^{\circ}\right]$. Until the 1950 s the majority of microbial metabolites were overlooked or merely regarded as waste products from primary metabolism. By contrast to a general set of primary metabolites, specialized metabolites are often specific to a restricted taxonomic range where they facilitate dedicated physiological, social or predatory functions [2]. Moreover, such metabolites have been found to possess a wide range of biological activities, making them useful for the development of antimicrobials, anticancer agents and immunosuppressants for pharmaceutical, agricultural and food manufacturing applications [3-6].

The majority of specialized metabolites result from metabolic pathways, each of which encoded by a suite of genes at the same chromosomal locus, generally known as biosynthetic gene clusters (BGCs). These BGCs are frequently 'silent' in common laboratory media, whereas their expression is triggered by specific environmental cues [7-9]. Recent developments in genomics and computational biology, hand in hand with a vastly increasing number of sequenced metagenomes and metatranscriptomes, have led to the discovery of thousands of BGCs $\left[10^{\bullet \bullet}, 11^{\bullet \bullet}\right]$.

Modular assembly lines such as PK synthases (PKS) and NRP synthetases (NRPS) constitute two of the most important and diverse classes of specialized metabolites that can theoretically code for a near infinite diversity of unique molecular architectures $\left[12,13,14^{\bullet \bullet}\right]$. Recent analyses based on retro-biosynthesis, that is, the computational breakdown of PK and NRP chemical molecules and reversal of their assembly lines to predict their parent PKS/NRPS BGCs, allow linking BGCs from publicly available databases to known natural products and define clusters encoding new products. Such efforts have shown that thousands of BGCs are likely to be responsible for the production of novel molecules $\left[10^{\bullet \bullet}\right]$.

To prevent replication of previous research and yet discover specialized metabolites from microbes with novel applicable biological activities, it is important to shift attention to environments and microbial phyla that have so far been largely neglected. Moreover, advanced bioinformatics analyses must be applied that can quickly assess the novelty of the gene clusters found and link them to predicted chemical structures and biological activities.

In this opinion paper, we highlight state-of-the-art developments regarding discovery, characterization 
Figure 1

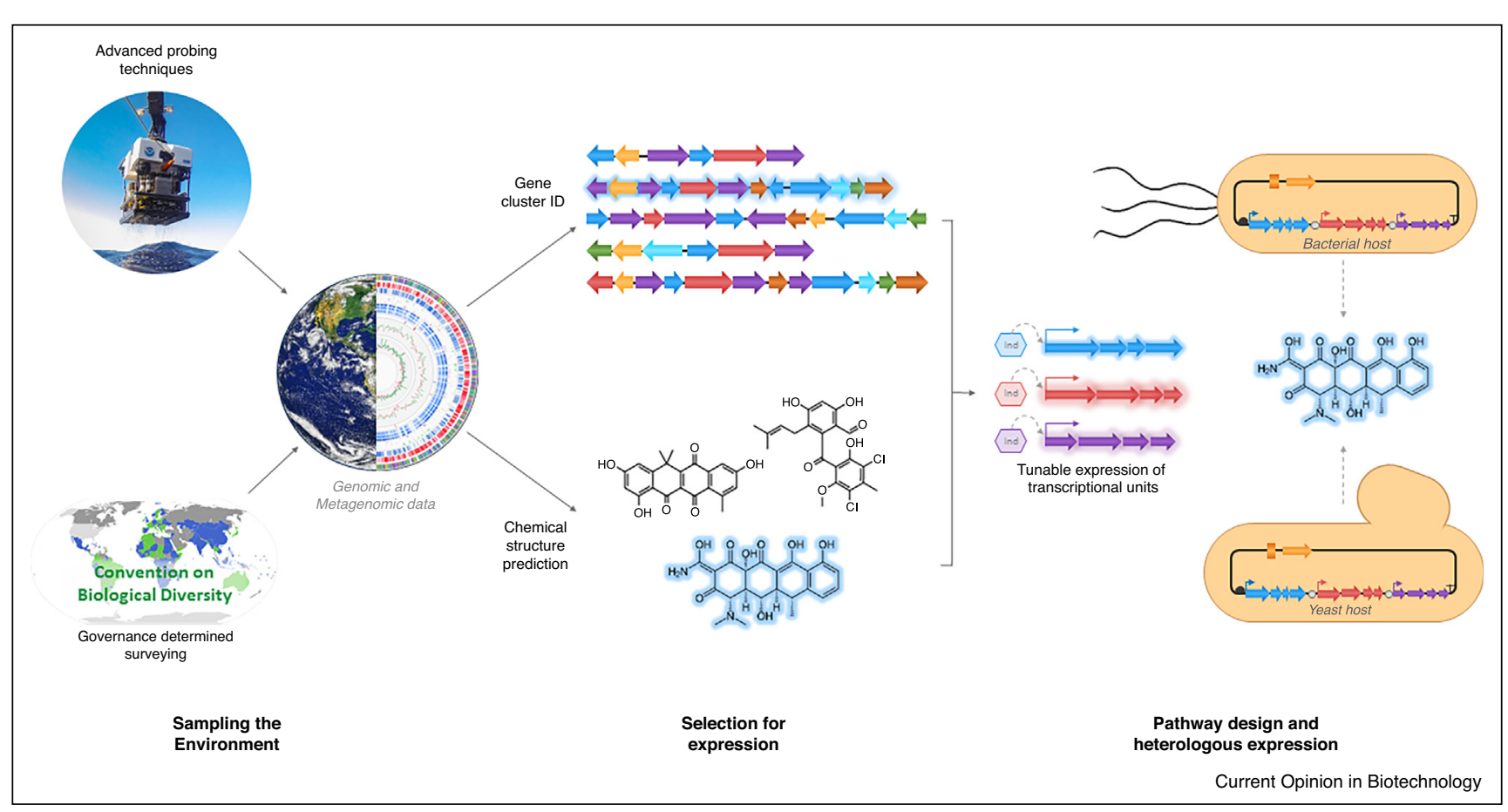

Approach for specialized metabolite discovery. Microbial specialized metabolites are of great value, and in order to boost their discovery, exploration of scarcely screened environments is key. Technological advances in sampling tools and techniques play an important role in allowing researchers to access such locations. At the same time, governmental constraints also dictate which regions will be favoured for exploration and exploitation of microbial bioactives. Newly developed computational methodologies enable mining of genomic and metagenomic data for detection of potentially new classes of biosynthetic gene clusters (BGCs). These algorithms are optimized to conduct identification of BGCs and predict their chemical structures, and are crucial to identify and select the most promising BGCs for further research and exploitation. The next step in unlocking and systematically exploiting these BGCs involves their controlled expression. Large DNA molecule manipulation involves assembly and cloning methods often based on homologous recombination mechanisms in both yeast and bacteria. Furthermore, advances in synthetic biology allowing customisation of transcriptional units' expression stoichiometry for production of complex chemicals, play an important role in the creation of automated production platforms.

and exploitation of microbial specialized metabolites, with a focus on PKS and NRPS. In addition, we identify environments, bioinformatics approaches and expression strategies that we consider most promising for future development of the field [Figure 1].

\section{Environmental sources of specialized metabolites}

Nature has provided mankind with numerous bioactive compounds for medical purposes for thousands of years, and even in modern times most drugs are derived from natural sources [15]. Bacteria and fungi that are responsible for the production of small bioactive molecules have been found in widely diverse environmental niches, such as soil, sediment and aquatic environments, either as freeliving microorganisms or in symbiosis with plants and animals $[15,16]$. Soil-dwelling cultivable Actinobacteria, and members of the genus Streptomyces in particular, have been in the limelight as proliferous sources of specialized bioactive metabolites, as witnessed by the discoveries of the antibiotics actinomycin, streptomycin and chloramphenicol in the 1940s, and the antiparasitic agent ivermectin $\left[17,18,1^{\circ}\right]$. Also soil-derived isolates from other bacterial genera, such as Bacillus [20] and Pseudomonas $\left[12,21^{\circ}\right]$ are traditionally rich sources of specialized metabolites. Interestingly, there appear to be important differences in biosynthetic potential between taxonomic groups within these genera, according to their ecological specializations $[5,22]$. Fungi, historically also mainly isolated from soils, represent a sometimes overlooked, but prolific source of bioactive molecules (e.g. antibiotics such as penicillin) [5,23]. A recently published study explored the environmental factors that drive changes in PKS and NRPS encoding BGC diversity across geographically distinct soil environments, and found changes in biosynthetic domain composition to correlate most consistently with variations in latitude [24].

However, cultivation-independent methods have shown that the uncultivated majority of the microorganisms encode many more BGCs (quantitatively and qualitatively) than the ones we know from isolates, a terra incognita with major potential for applications [4,5]. In addition, the use of these cultivation-independent 
methods shows that the traditional focus on Actinobacteria needs a shift towards other microorganisms such as marine fungi [25], Cyanobacteria [26,27], Proteobacteria [28] and the novel candidate phylum Tectomicrobia $\left[29,30^{\bullet \bullet}\right]$. For example, the latter, represented by a newly discovered uncultivated marine sponge symbiont genus Candidatus Entotheonella which has the genetic capacity to produce over 40 natural compounds and is widely distributed in taxonomically diverse sponges $\left[30^{\circ}\right]$. Other microbial taxa including Clostridium, Planctomycetes, Burkholderia and Xenorhabdus/Photorhabdus are also emerging important targets with high biosynthetic potential $[5,22]$.

Although the terrestrial environment is by no means exhausted of novel bioactive molecules, a recent large metagenomics study of the ocean water revealed that a stunning $90 \%$ of the genes detected at a depth of $600 \mathrm{~m} \mathrm{did}$ not have a match in public databases [31]. Although the ocean metagenome appeared to be rich in BGCs, we propose that the majority of BGCs in ocean water remains undetected as only the fraction $<3 \mu \mathrm{m}$ was considered in the aforementioned study, excluding small particles that are colonised by a community of microorganisms. These in turn are more likely to produce specialized metabolites of interest required for short-range molecular interactions. The same may be expected for marine sediments and biofilms (e.g. on macroalgae) that have been poorly investigated for their potential to produce specialized metabolites [32,33]. In addition, marine invertebrates display species-specific symbioses with microorganisms facilitated by unique metabolites, some of which may be valuable bioactive small molecules [34]. Particularly sponges, the biomass of which may be almost equally divided between host and symbionts, have been identified as one of the most promising natural source for future antibiotics $[35,36]$.

In addition to differences in the resource potential of particular natural environments, the governmental situation may dictate which regions will be favoured for exploration and exploitation of microbial bioactives. Compliance with the Nagoya Protocol requires the explorer to legally acquire any genetic resource, prove due diligence through traceability, risk assessment and risk mitigation procedures, and enable inspections by national authorities. Each signatory state may either determine its own access policy, or provide free access to its genetic resources and associated traditional knowledge (www.cbd.int/abs). However, concepts of biological diversity that are the foundation of the Nagoya Protocol are not directly applicable for microorganisms that do not abide the same patterns of endemism as plants and animals [37 $]$. For example, Streptomyces carpaticus strains isolated from coastal habitats in four different continents all produced the same cytotoxic specialized metabolite (Ikarugamycin) [38]; to a large degree, 'everything is everywhere' where the environment selects for the same molecular functions
[39]. In addition, structurally very similar polyketides have been obtained from bacterial symbionts from either insects or sponges [40]. Therefore, countries that have a more open attitude and lower administrative burden towards scientific exploration and commercial application of microbial specialized metabolites will likely be more attractive for scientists and industries.

\section{Rapid identification and prioritizing specialized metabolites}

In recent years, genome mining for BGCs has become a key approach for identification of new molecules and corresponding novel products. For compounds produced by PKSs and NRPSs, their biosynthetic pathways and product structures can be predicted using a range of computational tools and approaches [Table 1] [41-47].

The ability to detect potentially new classes of BGCs, including those prevailing in the uncultured majority of microorganisms, is a valuable endeavour as these will most likely code for molecules with new chemical scaffolds $\left[51,52^{\bullet}\right]$. Tools such as ClusterFinder $\left[11^{\bullet \bullet}, 53\right]$ and EvoMining [48] have been developed for this purpose. The former queries genome sequences for BGC-like regions based on the presence of broad Pfam protein domains associated with enzyme families commonly recycled in diverse specialized metabolic pathways. The latter exploits the notion that enzymes involved in specialized metabolism are paralogs of primary ones, which have undergone sequence and functional divergence, and utilizes phylogenetic analyses to detect these outliers [47,54]. Recent developments in high-throughput, single cell and long-read next-generation sequencing technologies are leading us to an era of fast, affordable sequencing and assembly of genomes from microbial isolates/consortia. Thus, it is becoming increasingly feasible to access culturable bacterial taxa and obtain highquality genomes from these strains, despite the presence of repetitive genomic regions such as those including BGCs encoding NRPS and PKS enzymes [22,47,54-57].

Moreover, through direct capture of environmental DNA from microbiomes of macroorganisms, metagenomics allows efficiently moving biosynthetic diversity from the environment into the drug discovery space [57]. PCR-based sequence tag approaches that screen metagenomic libraries for biosynthetic novelty are considered well established technologies $[4,57,58]$. However, despite being plagued by issues related to acquiring highly contiguous assemblies of BGCs, sequencing and assembly of environmental DNA by shotgun metagenomics constitutes a much more unbiased approach to profile biosynthetic diversity [22]. Whereas artificial long-read technologies offer valuable improvements in assembly quality $\left[59^{\circ}, 60,61\right]$, PK and NRP BGCs are usually still hard to assemble and often remain fragmented across multiple contigs. Nevertheless, contigs generated by De Bruijn 
Table 1

Tools for identification of BGCs.

\begin{tabular}{|c|c|c|}
\hline Tool & Approach & Reference \\
\hline antiSMASH & Identification or signature genomic and protein domains that are hallmarks of biosynthetic pathways. & [46] \\
\hline SMURF & Usually making use of profile Hidden Markov Models (HMM), BLAST and both general databases like & [41] \\
\hline PRISM & Pfam and specialized PKS/NRPS databases for annotation and protein identification. & [43] \\
\hline NP.searcher & & [45] \\
\hline CLUSEAN & & [44] \\
\hline EvoMining & $\begin{array}{l}\text { Exploits the notion that enzymes involved in specialized metabolism are paralogs of primary ones, } \\
\text { which have undergone sequence and functional divergence to acquire functions in specialized } \\
\text { metabolism. Utilizes phylogenetic mining to detect these outliers. }\end{array}$ & [48] \\
\hline ClusterFinder & $\begin{array}{l}\text { Queries genome sequences for BGC-like regions based on the presence of Pfam protein domain } \\
\text { frequencies associated with enzyme families that are indicative of diverse specialized metabolic } \\
\text { pathways. }\end{array}$ & {$\left[11^{\bullet \bullet}\right]$} \\
\hline GRAPE & $\begin{array}{l}\text { Retro-biosynthesis, that is, computational deconstruction of PK and NRP chemical structures to } \\
\text { predict their parent PKS/NRPS, producing assembly line monomers and tailoring enzymes. }\end{array}$ & [49] \\
\hline Bandage & $\begin{array}{l}\text { Tool for visualizing de Bruijn assembly graphs, allows for a deeper analysis of de novo assemblies } \\
\text { which is not accessible through study of individual contigs. }\end{array}$ & {$[50]$} \\
\hline
\end{tabular}

Graph assembly algorithms are not islands on their own, but in fact are connected to other contigs in an assembly graph. Although information contained in the assembly graph is lost in the way sequence assemblies are usually studied, the assembly graph files themselves can be analyzed with visualization software tools like Bandage [50]. By performing BLAST similarity searches on such a graph, one can often derive which BGC fragments belong to the same gene cluster. Based on this, clusters can potentially be reconstructed by finding the most plausible path through the assembly graph based on homology inference (as recently done for the bananamide BGC in a fragmented draft genome of Pseudomonas fluorescens BW11P2 [62] [Figure 2] [21 ${ }^{\circ}$ ]) or otherwise by designing primers to amplify and Sanger-sequence the gaps between the contigs. Alternatively, long read nanopore sequencing can also be used [63]. Additionally, binning metagenomes into metagenome-assembled genomes (MAGs) based on differential coverage and oligonucleotide frequencies, and subsequently re-assembling/finishing of high-quality MAGs allows increasing the contiguity of the assembly for particular organisms within a microbial community [64].

These and other computational methodologies are now making it possible to assemble many complete biosynthetic gene clusters from relatively complex metagenomes. The prediction of natural product structures from gene clusters is still challenging as deviations in gene order and enzyme modularity occur frequently [49], and predicting the regioselectivity of tailoring reactions is very complicated. Nevertheless, computational dereplication strategies based on sequence similarity [ $\left.1^{\bullet}\right]$ or retro-biosynthesis [49] make it possible to reliably identify BGCs that are likely to be involved in the production of novel chemical scaffolds. Moreover, target-based genome mining based on the detection of resistance genes within BGCs [65] makes it possible to pinpoint 'low-hanging fruits' that are likely to be responsible for the production of molecules that bind to cellular targets of interest, as the resistance genes often constitute paralogous copies of these molecular targets that are insensitive to the product of the BGC. Based on such and other criteria, at least a sub-set of BGCs can be intelligently shortlisted for expression studies.

\section{Heterologous expression strategies for specialized metabolites}

Biodiversity profiling of different environmental niches provides an outline of the phylogenetic composition of the corresponding communities, and demonstrates that uncultured species outnumber their cultured counterparts. Therefore, the quest to functionally express BGCs is currently the most urgent issue to unlock and exploit these gene clusters. However, this is not a straightforward undertaking. Firstly, because many BGCs are found in non-model organisms, often with rather distinct codon usage to general production hosts such as $E$. coli. Secondly, they are often encoded by clusters that can span over $100 \mathrm{~kb}$ of DNA, possibly including complex regulatory mechanisms [66]. Nevertheless, several methods have been developed allowing PKS and NRPS gene clusters to be successfully cloned and expressed in bacteria and fungi $\left[67-71,72^{\bullet \bullet}, 73,74\right]$.

DNA assembly methods, such as transformation-associated recombination (TAR) cloning are powerful tools for manipulating large DNA molecules. TAR makes use of homologous recombination in yeast and it has been successfully applied to clone and express the $73 \mathrm{~kb}$ gene cluster encoding the antibiotic taromycin A, originating from a marine actinomycete $\left[72^{\bullet \bullet}\right]$. Furthermore, a number of direct cloning methods allow integration of gene clusters at specific sites in the production host's chromosome, mainly via standard recombination methods. Direct cloning via Red/ET recombineering is based on E. coli linear plus linear homologous recombination [75], and has been successfully used to express large biosynthetic 
Figure 2

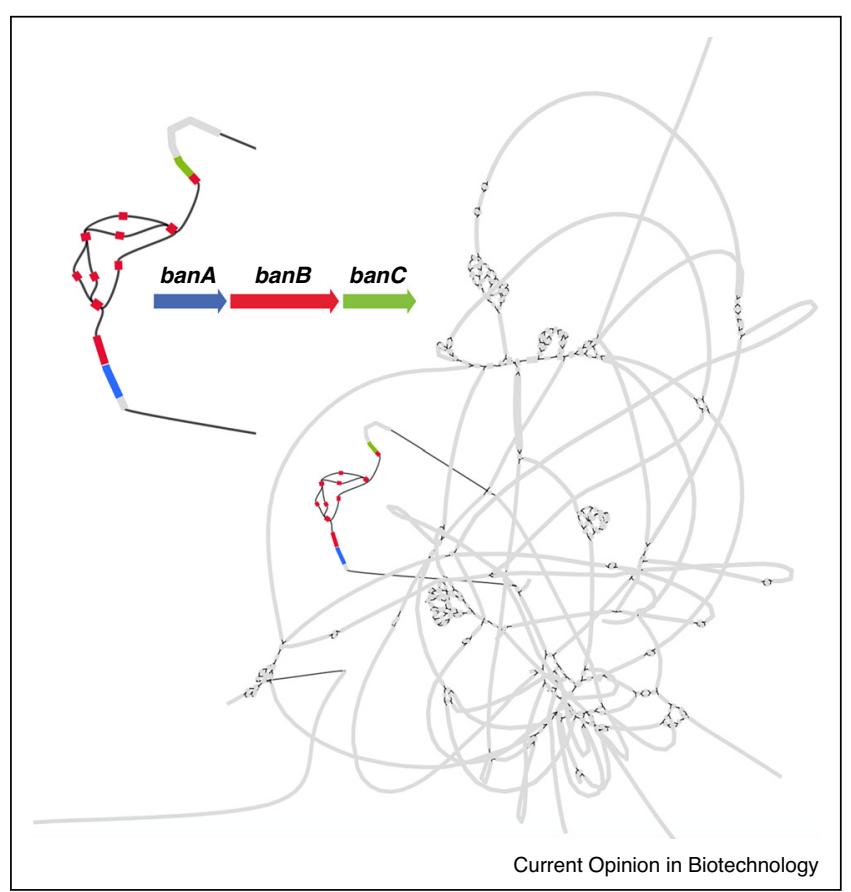

Assembly graph of a fragmented draft genome of Pseudomonas fluorescens BW11P2 [54], assembled by SPAdes, containing the reconstructed bananamide BGC. In the graph, the grey lines represent nodes (contigs) and black lines paths that represent possible connections between contigs. The upper left corner of the panel depicts a zoom-in visualization for the BLAST result of the genes in this BGC, blue, red and green represent the BLAST hits for gene banA, banB and banC correspondingly. Co-localization on the same node of banA and part of banB indicates proximity of these genes on the genome.

pathways such as the NPRS clusters coding for edeine $(48.3 \mathrm{~kb})$ and bacillomycin $(37.3 \mathrm{~kb})[70]$.

Advances in synthetic biology (including DNA construction tools, synthetic regulatory circuits and multiplexed genome engineering) enable the harnessing of metagenomic data for high-throughput molecular discovery, as well as pathway design for the production of complex chemicals $[66,74,76]$. In at least one instance, using a plug-and-play DNA assembly strategy to achieve full gene cluster refactoring in a single step manner has proved more effective than direct cloning and promoter insertion. This also made it possible to construct an automated platform with a high degree of flexibility for generating gene deletions or additions $\left[77^{\circ \bullet}\right]$. As a proof of principle for this approach, Luo and colleagues succeeded in expressing and characterizing a cryptic BCG encoding for the production of a polycyclic tretamate macrolactam PKS-NRPS hybrid $\left[77^{\circ \bullet}\right]$. With the continuous decrease in DNA synthesis cost, synthetic (codon-optimized) versions of many BGCs can be reconstructed in highthroughput using this technology.

One key issue that is difficult to address, especially for gene clusters for which the real molecular product is unknown beforehand, is that of cross-talk between the heterologously expressed pathway and the native pathways. A recent study by Zhang $e$ t al. showed that heterologous expression of the lyngbyatoxin gene cluster in three different streptomycete hosts lead to the generation of different natural product derivatives [78]. Because small variations in chemical structure can have a major impact on biological activity, expression studies in multiple hosts (or multiple versions of the same hosts with different native BGCs knocked out using, e.g. CRISPR/Cas) are required to ascertain the true product of a synthetically reconstructed $\mathrm{BGC}$ for which the native product is unknown. In the more distant future, it might be worth considering to design 'orthogonal' heterologous expression strategies that isolate the heterologous pathway from native metabolism, for example, through compartmentalization $[79,80]$.

\section{Outlook}

Microbial specialized metabolites are a vast and exceptional resource that may contribute to solving the current antibiotic resistance crisis $\left[19^{\bullet}, 81,82\right]$. Based on several technological advances, it is now possible to reach and sample the most difficult-to-access places on Earth. Exploration of scarcely touched environments in combination with the revolutionary developments in metagenomics and computational biology has already led to an 
explosion in the number of known BGC sequences. Our greatest current challenge is to systematically use these sequences for the production of specialized metabolites and the discovery of their biological functions. Notwithstanding, we have witnessed a growing number of success cases in the past decade, including the activation or heterologous expression of cognate BGCs from nonmodel organisms leading to the successful production of several previously unknown secondary metabolites. Ultimately, the implementation of multi-omics approaches that combine ecological insights with state-of-the-art computational and molecular genomics developments will lead to deep understanding and more efficient exploitation of microbial specialized metabolism.

\section{Conflict of interest}

None.

\section{Acknowledgements}

CL was funded by NWO-VLAG grant Mare incognita, MHM was supported by VENI grant 863.15 .002 from The Netherlands Organization for Scientific Research (NWO), JvdO is supported by the Netherlands Organization for Scientific Research (NWO) by a TOP grant (714.015.001) and DS acknowledges funding from the European Union's Horizon 2020 research and innovation program under Grant Agreement No. 679849 (SponGES).

\section{References and recommended reading}

Papers of particular interest, published within the period of review, have been highlighted as:

- of special interest

•• of outstanding interest

1. Medema MH: The Minimum Information about a Biosynthetic

- Gene cluster (MIBiG) specification. Nat Chem Biol 2015:625631. revised ve.

The authors propose the creation of an information standard, the Minimum Information about a Biosynthetic Gene Cluster (MIBiG), to facilitate a systematic access to data on biosynthetic gene clusters.

2. Hartmann T: The lost origin of chemical ecology in the late 19th century. Proc Natl Acad Sci U S A 2008, 105:4541-4546.

3. Medema MH, Fischbach MA: HHS public access. Nat Chem Biol 2016, 11:639-648.

4. Milshteyn A, Schneider JS, Brady SF: Mining the metabiome: identifying novel natural products from microbial communities. Chem Biol 2014, 21:1211-1223.

5. Challinor VL, Bode HB: Bioactive natural products from novel microbial sources. 2015, 1354:82-97.

6. Harvey AL, Edrada-Ebel R, Quinn RJ: The re-emergence of natural products for drug discovery in the genomics era. Nat Rev Drug Discov 2015, 14:111-129.

7. Van der Meij A, Worsley SF, Hutchings MI, Van Wezel GP: Chemical ecology of antibiotic production by actinomycetes. FEMS Microbiol Rev 2017, 41:392-416.

8. Abdelmohsen UR, Grkovic T, Balasubramanian S, Kamel MS, Quinn RJ, Hentschel U: Elicitation of secondary metabolism in actinomycetes. Biotechnol Adv 2015, 33:798-811.

9. Rutledge PJ, Challis GL: Discovery of microbial natural products by activation of silent biosynthetic gene clusters. Nat Rev Microbiol 2015, 13:509-523.

10. Dejong CA, Chen GM, Li H, Johnston CW, Edwards MR, Rees PN,

-. Skinnider MA, Webster ALH, Magarvey NA: Polyketide and nonribosomal peptide retro-biosynthesis and global gene cluster matching. Nat Chem Biol 2016, 12:1007-1014.
In this study a retro-biosynthetic program is created to link biosynthetic gene clusters to known chemical molecules. This approach aims at clarifying which gene clusters encode previously isolated chemical compounds.

11. Cimermancic $\mathrm{P}$, Medema MH, Claesen J, Kurita K, Wieland

- Brown LC, Mavrommatis K, Pati A, Godfrey PA, Koehrsen M, Clardy $\mathrm{J}$ et al.: Insights into secondary metabolism from a global analysis of prokaryotic biosynthetic gene clusters. Cell 2014, 158:412-421.

A novel algorithm (ClusterFinder) was created which identifies potential biosynthetic gene clusters in microbial sequencing data. The approach ultimately led to the characterization of the most prominent family of BGCs in sequence databases.

12. Helfrich EJN: Biosynthesis of polyketides by trans-AT polyketide synthases. Nat Prod Rep 2016 http://dx.doi.org/ 10.1039/C5NP00125K.

13. Doroghazi JR, Metcalf WW: Comparative genomics of actinomycetes with a focus on natural product biosynthetic genes. BMC Genomics 2013, 14:611.

14. Rees P, Johnston CW, Skinnider MA, Dejong CA, Rees PN,

-. Chen GM, Walker CG, French S, Brown ED, Bérdy J et al:: Assembly and clustering of natural antibiotics guides target identification. Nat Chem Biol 2018 http://dx.doi.org/10.1038/ nchembio.2018.

This study explores the direct mining of biosynthetic gene clusters and associated antimicrobial resistance determinants to uncover specialized metabolites with previously unknown modes of action.

15. Newman DJ, Cragg GM: Natural products as sources of new drugs over the $\mathbf{3 0}$ years from 1981 to 2010. J Nat Prod 2012, 75:311-335.

16. Pi B, Yu D, Dai F, Song X, Zhu C, Li H: A genomics based discovery of secondary metabolite biosynthetic gene clusters in Aspergillus ustus. PLoS One 2015 http://dx.doi.org/10.1371/ journal.pone.0116089.

17. Barka EA, Vatsa P, Sanchez L, Gaveau-vaillant N, Jacquard C, Klenk H-P, Clément C, Ouhdouch Y, van Wezel GP: Taxonomy, physiology, and natural products of Actinobacteria. Microbiol Mol Biol Rev 2016, 80:1-43.

18. Campbell WC, Fisher MH, Stapley EO, Albers-Schönberg G Jacob TA: Ivermectin: a potent new antiparasitic agent. Science (80-) 1983, 221:823-828.

19. Ling LL, Schneider T, Peoples AJ, Spoering AL, Engels I,

- Conlon BP, Mueller A, Hughes DE, Epstein S, Jones M et al.: A new antibiotic kills pathogens without detectable resistance. Nature 2015, 517:455-459.

A screen of previously uncultured microorganisms using an iChip multichannel diffusion growth chamber yielded the antibiotic teixobactin which inhibits cell wall synthesis. Resistant mutants of relevant pathogens were not obtained.

20. Zhao X, Kuipers OP: Identification and classification of known and putative antimicrobial compounds produced by a wide variety of Bacillales species. BMC Genomics $2016 \mathrm{http}: / / \mathrm{dx}$.doi. org/10.1186/s12864-016-3224-y.

21. Nguyen DD, Melnik AV, Koyama N, Lu X, Schorn M, Fang J,

- Aguinaldo K, Lincecum TL, Ghequire MGK, Carrion VJ et al: Indexing the Pseudomonas specialized metabolome enabled the discovery of poaeamide B and the bananamides. Nat Microbiol 2016, 2:16197.

The authors map the diversity of the Pseudomonas metabolome, based on mass-spectrometry molecular networking. This led to the discovery of four molecules, and shows how the approach can be used to annotate data from other studies.

22. Tracanna V, de Jong A, Medema MH, Kuipers OP: Mining prokaryotes for antimicrobial compounds: from diversity to function. FEMS Microbiol Rev 2017, 41:417-429.

23. Alberti F, Foster GD, Bailey AM: Natural products from filamentous fungi and production by heterologous expression. Appl Microbiol Biotechnol 2017, 101:493-500.

24. Lemetre C, Maniko J, Charlop-Powers Z, Sparrow B, Lowe AJ, Brady SF: Bacterial natural product biosynthetic domain composition in soil correlates with changes in latitude on a continent-wide scale. Proc Natl Acad Sci 2017, 114:201710262. 
25. Nicoletti R, Trincone A: Bioactive compounds produced by strains of Penicillium and Talaromyces of marine origin. Mar Drugs 2016, 14:1-35 Article 37.

26. Banik JJ, Brady SF: Recent application of metagenomic approaches toward the discovery of antimicrobials and other bioactive small molecules. Curr Opin Microbiol 2010, 13: 603-609.

27. Long PF, Dunlap WC, Battershill CN, Jaspars M: Shotgun cloning and heterologous expression of the patellamide gene cluster as a strategy to achieving sustained metabolite production. ChemBioChem 2005, 6:1760-1765.

28. Desriac F, Jégou C, Balnois E, Brillet B, Le Chevalier P, Fleury Y: Antimicrobial peptides from marine proteobacteria. Mar Drugs 2013, 11:3632-3660

29. Martins A, Vieira H, Gaspar H, Santos S: Marketed marine natural products in the pharmaceutical and cosmeceutical industries: tips for success. Mar Drugs 2014, 12:1066-1101.

30. Wilson MC, Mori T, Rückert C, Uria AR, Helf MJ, Takada K,

$\bullet \quad$ Gernert C, Steffens UAE, Heycke N, Schmitt S et al.: An environmental bacterial taxon with a large and distinct metabolic repertoire. Nature 2014, 506:58-62.

In this study single cell analysis and metagenomics are used to uncover and characterize marine sponge bacteria symbionts which possess distinct biosynthetic gene clusters. The authors propose the candidate phylum "Tectomicrobia", with unique and pronounced specialized metabolism.

31. Sunagawa S, Coelho LP, Chaffron S, Kultima JR, Labadie K, Salazar G, Djahanschiri B, Zeller G, Mende DR, Alberti A et al.: Structure and function of the global ocean microbiome. Science (80-) 2015, 348:1261359.

32. Patin NV, Schorn M, Aguinaldo K, Lincecum T, Moore BS, Jensen PR: Effects of actinomycete secondary metabolites on sediment microbial communities. Appl Environ Microbiol 2017, 83:1-16.

33. Niederdorfer R, Besemer K, Battin TJ, Peter H: Ecological strategies and metabolic trade-offs of complex environmental biofilms. npj Biofilms Microbiomes 2017, 3:21.

34. Blunt JW, Copp BR, Keyzers RA, Munro MHG, Prinsep MR: Marine natural products. Nat Prod Rep 2017, 34:235-294.

35. Fortman JL, Mukhopadhyay A: The future of antibiotics: emerging technologies and stewardship. Trends Microbiol 2017, 24:515-517.

36. Sipkema D: Marine biotechnology: diving deeper for drugs Microb Biotechnol 2017, 10:7-8.

37. Overmann J, Scholz AH: Microbiological research under the

- $\quad$ nagoya protocol: facts and fiction. Trends Microbiol 2017. 25:85-88.

The authors comment on the inapplicability of the Nagoya Protocol to the field of microbiology, as the concepts of biodiversity it is based on do not correctly describe microbial communities.

38. Overmann J: Significance and future role of microbial resource centers. Syst Appl Microbiol 2015, 38:258-265.

39. De Wit R, Bouvier T: "Everything is everywhere, but, the environment selects"; what did Baas Becking and Beijerinck really say? Environ Microbiol 2006, 8:755-758.

40. Piel J, Hui D, Wen G, Butzke D, Platzer M, Fusetani N, Matsunaga S: Antitumor polyketide biosynthesis by an uncultivated bacterial symbiont of the marine sponge Theonella swinhoei. Proc Natl Acad Sci U S A 2004, 101: 16222-16227.

41. Khaldi N, Seifuddin FT, Turner G, Haft D, Nierman WC, Wolfe KH, Fedorova ND: SMURF: genomic mapping of fungal secondary metabolite clusters. Fungal Genet Biol 2010, 47:736-741.

42. Starcevic A, Zucko J, Simunkovic J, Long PF, Cullum J, Hranueli D: ClustScan: an integrated program package for the semiautomatic annotation of modular biosynthetic gene clusters and in silico prediction of novel chemical structures. Nucleic Acids Res 2008, 36:6882-6892.
43. Skinnider MA, Dejong CA, Rees PN, Johnston CW, Li H, Webster ALH, Wyatt MA, Magarvey NA: Genomes to natural products PRediction Informatics for Secondary Metabolomes (PRISM). Nucleic Acids Res 2015, 43:9645-9662.

44. Weber T, Rausch C, Lopez P, Hoof I, Gaykova V, Huson DH, Wohlleben W: CLUSEAN: a computer-based framework for the automated analysis of bacterial secondary metabolite biosynthetic gene clusters. J Biotechnol 2009, 140:13-17.

45. Li MHT, Ung PMU, Zajkowski J, Garneau-Tsodikova S, Sherman $\mathrm{DH}$ : Automated genome mining for natural products. BMC Bioinformatics 2009, 10:1-10.

46. Weber T, Blin K, Duddela S, Krug D, Kim HU, Bruccoleri R, Lee SY Fischbach MA, Müller R, Wohlleben W et al.: AntiSMASH 3.0-A comprehensive resource for the genome mining of biosynthetic gene clusters. Nucleic Acids Res 2015, 43: W237-W243.

47. Medema $\mathrm{MH}$, Fischbach MA: Computational approaches to natural product discovery. Nat Chem Biol 2016, 11:639-648.

48. Cruz-Morales P, Kopp JF, Martínez-Guerrero C, Yáñez-Guerra LA Selem-Mojica N, Ramos-Aboites H, Feldmann J, BaronaGómez F: Phylogenomic analysis of natural products biosynthetic gene clusters allows discovery of arseno-organic metabolites in model Streptomycetes. Genome Biol Evol 2016 8:1906-1916.

49. Dejong CA, Chen GM, Li H, Johnston CW, Edwards MR, Rees PN, Skinnider MA, Webster ALH, Magarvey NA: Polyketide and nonribosomal peptide retro-biosynthesis and global gene cluster matching. Nat Chem Biol 2016 http://dx.doi.org/10.1038/ nchembio.2188.

50. Wick RR, Schultz MB, Zobel J, Holt KE: Bandage: interactive visualization of de novo genome assemblies. Bioinformatics $2015,31: 3350-3352$.

51. Fischbach MA, Walsh CT: Antibiotics for emerging pathogens Science (80-) 2009, 325:1089-1093.

52. Rinke C, Schwientek P, Sczyrba A, Ivanova NN, Anderson IJ,

- Cheng J, Darling A, Malfatti S, Swan BK, Gies EA et al.: Insights into the phylogeny and coding potential of microbial dark matter. Nature 2013, 499:431-437.

Here single cell genomics is applied to target and sequence archaeal and bacterial cells from diverse habitats belonging to the 'microbial dark matter'. As a result, two new superphyla are proposed.

53. Punta M, Coggill PC, Eberhardt RY, Mistry J, Tate J, Boursnell C, Pang N, Forslund K, Ceric G, Clements J et al: The Pfam protein families database. Nucleic Acids Res 2012, 40:290-301.

54. Ziemert N, Alanjary M, Weber T: The evolution of genome mining in microbes - a review. Nat Prod Rep 2016, 33:988-1005.

55. Klassen JL, Currie CR: Gene fragmentation in bacterial draft genomes: extent, consequences and mitigation. BMC Genomics 2012, 13:14.

56. Epstein SS: The phenomenon of microbial uncultivability. Curr Opin Microbiol 2013, 16:636-642.

57. Charlop-powers Z, Milshteyn A, Brady SF: Metagenomic small molecule discovery methods. Curr Opin Microbiol 2014, 19:70 75.

58. Charlop-Powers Z, Pregitzer CC, Lemetre C, Ternei MA, Maniko J Hover BM, Calle PY, McGuire KL, Garbarino J, Forgione HM et al.: Urban park soil microbiomes are a rich reservoir of natural product biosynthetic diversity. Proc Natl Acad Sci U S A 2016, 113:14811-14816.

59. White RA, Bottos EM, Roy Chowdhury T, Zucker JD, Brislawn CJ, - Nicora CD, Fansler SJ, Glaesemann KR, Glass K, Jansson JK: Moleculo long-read sequencing facilitates assembly and genomic binning from complex soil metagenomes. mSystems 2016, 1 e00045-16.

Moleculo synthetic long-read sequencing technology is here applied in soil samples. Using this technique yielded raw reads of sizes over $10 \mathrm{~kb}$ and the first complete genome assembled from a soil metagenome.

60. Kuleshov V, Jiang C, Zhou W, Jahanbani F, Batzoglou S, Snyder M: Synthetic long-read sequencing reveals 
intraspecies diversity in the human microbiome. Nat Biotechnol 2015:34.

61. Moss E, Bishara A, Tkachenko E, Kang JB, Andermann TM, Wood C, Handy C, Ji H, Batzoglou S, Bhatt AS: De novo assembly of microbial genomes from human gut metagenomes using barcoded short read sequences. bioRxiv $2017 \mathrm{http} / / / \mathrm{dx}$.doi.org/ $10.1101 / 125211$.

62. Nguyen DD, Melnik AV, Koyama N, Lu X, Schorn M, Fang J, Aguinaldo K, Lincecum TL Jr, Ghequire MGK, Carrion VJ et al.: Indexing the Pseudomonas specialized metabolome enabled the discovery of poaeamide B and the bananamides. Nat Microbiol 2016, 2:16197.

63. Loman NJ, Quick J, Simpson JT: A complete bacterial genome assembled de novo using only nanopore sequencing data. Nat Meth 2015, 12:733-735.

64. Anantharaman K, Brown CT, Hug LA, Sharon I, Castelle CJ, Probst AJ, Thomas BC, Singh A, Wilkins MJ, Karaoz U et al.: Thousands of microbial genomes shed light on interconnected biogeochemical processes in an aquifer system. Nat Commun 2016, 7:13219.

65. Alanjary M, Kronmiller B, Adamek M, Blin K, Weber T, Huson D, Philmus B, Ziemert N: The Antibiotic Resistant Target Seeker (ARTS), an exploration engine for antibiotic cluster prioritization and novel drug target discovery. Nucleic Acids Res 2017, 22:43-51.

66. Smanski MJ, Zhou H, Claesen J, Shen B, Fischbach MA, Voigt CA Synthetic biology to access and expand nature's chemical diversity. Nat Rev Microbiol 2016, 14:135-149.

67. Du D, Wang L, Tian Y, Liu H, Tan H, Niu G: Genome engineering and direct cloning of antibiotic gene clusters via phage $\phi B T 1$ integrase-mediated site-specific recombination in Streptomyces. Sci Rep 2015, 5:8740

68. Ishiuchi K, Nakazawa T, Ookuma T, Sugimoto S, Sato M, Tsunematsu $\mathrm{Y}$, Ishikawa N, Noguchi H, Hotta K, Moriya H et al.: Establishing a new methodology for genome mining and biosynthesis of polyketides and peptides through yeast molecular genetics. ChemBioChem 2012, 13:846-854.

69. Chiang Y-M, Oakley CE, Ahuja M, Entwistle R, Schultz A, Chang SL, Sung CT, Wang CCC, Oakley BR: An efficient system for heterologous expression of secondary metabolite genes in Aspergillus nidulans. J Am Chem Soc 2013, 135:7720-7731.

70. Liu Q, Shen Q, Bian X, Chen H, Fu J, Wang H, Lei P, Guo Z, Chen W, Li D et al.: Simple and rapid direct cloning and heterologous expression of natural product biosynthetic gene cluster in Bacillus subtilis via Red/ET recombineering. Sci Rep 2016, 6:34623.

71. Tu Q, Herrmann J, Hu S, Raju R, Bian X: Genetic engineering and heterologous expression of the disorazol biosynthetic gene cluster via Red/ET recombineering. Nat Pub/ Gr 2016 http://dx. doi.org/10.1038/srep21066.

72. Yamanaka K, Reynolds KA, Kersten RD, Ryan KS, Gonzalez DJ,

-• Nizet V, Dorrestein PC, Moore BS: Direct cloning and refactoring of a silent lipopeptide biosynthetic gene cluster yields the antibiotic taromycin A. Proc Natl Acad Sci U S A 2014, 111:1957-1962.

In this study the yeast based transformation-associated recombination method was used to directly clone, refactor and heterologous express a $67 \mathrm{~kb}$ non ribosomal peptide synthase gene cluster from a marine actinomycete.

73. Shao Z, Zhao H, Zhao H: DNA assembler, an in vivo genetic method for rapid construction of biochemical pathways. Nucleic Acids Res 2009, 37:1-10.

74. Luo Y, Enghiad B, Zhao H: New tools for reconstruction and heterologous expression of natural product biosynthetic gene clusters. Nat Prod Rep 2016, 0:1-9.

75. Muyrers JPP, Zhang Y, Stewart AF: Techniques: recombinogenic engineering - new options for cloning and manipulating DNA. Trends Biochem Sci 2001, 26:325-331.

76. Reports NP, Andrews S, Scienc I, Rese P: The generation of "unNatural" products: synthetic biology meets synthetic chemistry. Nat Prod Rep 2012 http://dx.doi.org/10.1039/ c2np00001f.

77. Luo Y, Huang H, Liang J, Wang M, Lu L, Shao Z, Cobb RE, Zhao H:

-. Activation and characterization of a cryptic polycyclic tetramate macrolactam biosynthetic gene cluster. Nat Commun 2013, 4:2894.

Here a cryptic polyclic tetramate macrolactam biosynthetic gene cluster is activated by use of a synthetic biology approach. The hybrid polyketide synthase - nonribosomal peptide synthetase is further characterized by designing a series of gene deletion constructs.

78. Zhang L, Hoshino S, Awakawa T, Wakimoto T, Abe I: Structural diversification of lyngbyatoxin A by host-dependent heterologous expression of the tleABC biosynthetic gene cluster. ChemBioChem 2016, 17:1407-1411.

79. Chen $\mathrm{AH}$, Silver PA: Designing biological compartmentalization. Trends Cell Biol 2012, 22:662-670.

80. Agapakis CM, Boyle PM, Silver PA: Natural strategies for the spatial optimization of metabolism in synthetic biology. Nat Chem Biol 2012, 8:527-535.

81. Schaik $W$ Van: The human gut resistome. Phil Trans $R$ Soc $B$ 2015:370.

82. Laxminarayan R, Duse A, Wattal C, Zaidi AKM, Wertheim HFL, Sumpradit N, Vlieghe E, Hara GL, Gould IM, Goossens H et al.: Antibiotic resistance - the need for global solutions. Lancet Infect Dis 2013, 13:1057-1098. 